\title{
Peptide mimics of a carbohydrate-associated epitope expressed by cancer cells: Identification of vaccine candidates
}

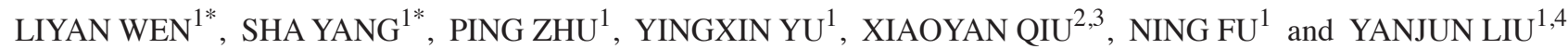 \\ ${ }^{1}$ Department of Immunology, School of Basic Medical Science, Southern Medical University, Guangzhou, Guangdong 510515; \\ ${ }^{2}$ Department of Immunology, School of Basic Medical Sciences, Peking University Health Science Center; \\ ${ }^{3}$ Key Laboratory of Medical Immunology, Ministry of Health, Beijing 100191; \\ ${ }^{4}$ Guangdong Province Key Laboratory of Proteomics, Southern Medical University, \\ Guangzhou, Guangdong 510515, P.R. China
}

Received October 8, 2015; Accepted August 30, 2016

DOI: $10.3892 / \mathrm{mmr} .2016 .5863$

\begin{abstract}
Cancer-associated antigen 215 (CA215) is an immunoglobulin molecule expressed by numerous tumor types. Membrane-bound and soluble CA215 have been detected in the majority of cancer cells and rarely identified in normal tissues. In addition, CA215C is a carbohydrate-associated epitope in the variable region of CA215, which is specifically recognized by the monoclonal antibody, RP215. However, CA215C is not a suitable vaccine candidate as it is a thymus-independent antigen. In the present study, RP215 was used as a target to screen short peptide mimics of CA215C from a phage display peptide library. Following three rounds of screening, 30 positive phage clones that specifically bound to RP215 were identified and sequenced. The result of amino-acid sequence analysis revealed five conserved sequence groups for seventeen of the positive phage clones. The sequences of phage clones 2, 13 and 42 were selected for peptide synthesis and binding analysis. The synthetic peptides R2 and R42 specifically bound RP215. Antisera from mice immunized with R2-BSA or R42-BSA
\end{abstract}

Correspondence to: Dr Yanjun Liu, Department of Immunology, School of Basic Medical Science, Southern Medical University, 1023-1063 Shatainan Road, Guangzhou, Guangdong 510515, P.R. China

E-mail: yanjun@smu.edu.cn

*Contributed equally

Abbreviations: Ig, immunoglobulin; TI-Ag, thymus-independent antigen; TD-Ag, thymus-dependent antigen; mAb, monoclonal antibody; IHC, immunohistochemistry; HRP, horseradish peroxidase; TMB, tetramethylbenzidine; V region, variable region; ELISA, enzyme-linked immunosorbent assay; PBS, phosphate buffered saline; PBST, PBS containing 0.1\% Tween-20; BSA, bovine serum albumin; MES, 2-(N-Morpholino) ethansulfonic acid monohydrate; H\&E, hematoxylin-eosin; NC, negative control

Key words: RP215, carbohydrate-associated epitope, peptide mimics, phage display library, cancer-associated antigen 215 bound purified CA215C and innate CA215C expressed on human hepatic and rectal carcinoma tissues, as demonstrated by immunohistochemistry. Furthermore, R2-BSA and R42-BSA antisera inhibited RP215 binding to cancer tissues. These results revealed that R2-BSA and R42-BSA antisera had similar characteristics to RP215 and that the synthetic peptides R2 and R42 may mimic the CA215C epitope. R2 and $\mathrm{R} 42$ peptides may therefore have potential for development into a tumor vaccine.

\section{Introduction}

Immunoglobulins (Igs) are important immunological molecules and were considered to be specific products of $\mathrm{B}$ lymphocytes. However, in 2003, Qiu et al (1) demonstrated that Ig may be derived from and expressed by human cancer cells of epithelial origin. Ig derived from non-B cells, including human epithelial cancer cells and certain normal epithelial cells, is referred to as non-B Ig. The existence of non-B Ig has now been confirmed by further studies, which in addition have revealed that cancer-derived Ig contributes to the malignization, migration and proliferation of cancer cells $(2,3)$.

Lee et al (4) generated the monoclonal antibody (mAb), RP215 (hybridoma, HB10095; US patent no. US5650291) and demonstrated that it bound a certain molecule in numerous cancer cell lines, as well as serum and tissue sections from patients with various cancers, including ovarian, cervical, endometrial, breast, stomach and colon cancer; however, RP215 did not bind to healthy cells (5-8). The molecule recognized by RP215 was named cancer-associated antigen 215 (CA215). As it is markedly homologous to normal human IgG heavy chain, CA215 was considered a non-B Ig (4). CA215 may be an important pan-biomarker for numerous human cancers and a potential target for clinical monitoring. Lee et al $(4,9)$ further confirmed that the epitope recognized by RP215 was a carbohydrate-associated epitope on CA215 (CA215C). Using RP215 to target CA215 through CA215C inhibited cancer cell proliferation, induced apoptosis in vitro and inhibited the growth of human tumor cells in nude mice $(10,11)$. Therefore, RP215 may be a potential therapeutic 
agent for the treatment of cancer, and has been developed as a therapeutic humanized antibody (10). The specific binding between RP215 and CA215C suggests that CA215C may be a potential vaccine candidate to induce active immunity against cancer. However, as CA215C is a carbohydrate-associated epitope(s) with weak immunogenicity, it is not suitable as a vaccine candidate. As thymus-independent antigens (TI-Ag), carbohydrate-associated epitopes do not induce specific cellular immune responses and effective secondary antibody responses.

The aim of the present study was to develop CA215C epitopes into therapeutic tumor vaccine candidates by enabling them to induce effective adaptive immune responses. In the present study, peptide mimics of CA215C epitopes were screened from a random phage display peptide library using the mAb RP215 as the target molecule. The synthetic peptide mimics were recognized by RP215 specifically, and effectively induced antisera/antibody production in mice, specifically targeting CA215 on human cancer tissues, suggesting that these peptide mimics may be potential candidates for tumor vaccine development.

\section{Materials and methods}

Reagents. The Ph.D. ${ }^{\text {TM }}-12$ phage display library was purchased from New England Biolabs Ltd. (Hitchen, UK). The mAb RP215 and purified CA215 were obtained from Professor GregoryLee at the University of British Columbia (Vancouver, BC, Canada). The mAbs 4D11 (12) and 2H4 (13) were prepared in our laboratory as previously described. Goat anti-human IgG polyclonal antibody (catalog no. 05204005) and horseradish peroxidase (HRP)-conjugated goat anti-human IgG (catalog no. 05204001) were purchased from Multi Sciences (Lianke) Biotech Co., Ltd. (Hangzhou, China). Peroxidase AffiniPure goat anti-mouse IgG (catalog no. 115-035-003) was purchased from Jackson ImmunoResearch Laboratories Co., Inc. (West Grove, PA, USA). HRP-labeled anti-M13 (catalog no. 27942101) was obtained from GE Healthcare Life Sciences (Chalfont, UK). Normal human IgG (catalog no. VGNHH-12000C) was purchased from Multi Science (Lianke) Biotech Co., Ltd. Normal mouse IgG (catalog no. CDF01-100UG) was purchased from EMD Millipore (Billerica, MA, USA). Streptavidin-HRP was purchased from Beijing Dingguochangsheng Biotechnology Co., Ltd. (Beijing, China). The synthetic peptides were produced by Hybio Pharmaceutical Co., Ltd. (Shenzhen, China). The enhanced HRP-diaminobenzidine (DAB) chromogenic substrate kit was purchased from Tiangen Biotech Co., Ltd. (Beijing, China) and the endogenous biotin-avidin blocking kit was purchased from Shanghai YuanMu Biological Technology Co., Ltd. (Shanghai, China). All of the chemicals used in this study were obtained from Guangzhou Jinhuada Chemical Reagent Co., Ltd. (Guangzhou, China), unless otherwise stated.

Mice. A total of 15 female BALB/cmice (age, 6-8 weeks) were purchased from the Animal Center of Southern Medical University (Guangzhou, China). Mice were housed at a temperature of $18-22^{\circ} \mathrm{C}$ and $50-60 \%$ humidity under a $10 / 14-\mathrm{h}$ light/dark cycle and with free access to food and water. All procedures were conducted in accordance with the National Institutes of Health Guide for the Care and Use of Laboratory Animals, and with the Guidelines for the Care and Use of Animals established by the Animal Care and Use Committee of the Southern Medical University. The present study was approved by the Ethical Committee of Southern Medical University.

Tissue samples. Tissue samples, including hepatic and rectal carcinoma tissues, were collected from the Department of Pathology of NanFang Hospital (Guangzhou, China). Written informed consent was obtained from patients or their families. All procedures were performed with the approval of the Institutional Review Board of Southern Medical University. For immunohistochemistry (IHC), biopsy tissues had been fixed immediately in $10 \%$ buffered formalin, and $24 \mathrm{~h}$ later were dehydrated in increasing concentrations of alcohol and embedded in paraffin. Serial $5-\mu \mathrm{m}$ thick sections were then cut using a microtome.

\section{Screening and characterization of phage clones that mimic CA215C}

Screening of the phage display peptides. Phage clones that mimicked CA215C were screened from a 12-mer linear phage display peptide library based on an antigen-antibody reaction described previously (14). Panning of the phage display library was performed according to the manufacturer's protocol. Briefly, RP215 diluted to $5 \mu \mathrm{g} / \mathrm{ml}$ with $0.1 \mathrm{M} \mathrm{NaHCO}_{3}$ (pH 8.6) was coated onto 96-well plates as a target protein. A total of $5 \times 10^{9}$ phage particles were added to the wells and incubated at room temperature for $1 \mathrm{~h}$. The non-binding phage clones were washed off with phosphate buffered saline (PBS) containing 0.1\% Tween-20 (PBST). The bound phage clones were eluted using $0.2 \mathrm{M}$ glycine- $\mathrm{HCl}$ buffer ( $\mathrm{pH}$ 2.2) and were immediately neutralized with $1 \mathrm{M}$ Tris- $\mathrm{HCl}$ ( $\mathrm{pH}$ 9.1). The eluted phage clones were titrated on isopropyl $\beta$-D-1-thiogalactopyranoside (IPTG)/X-gal agar plates, amplified with host strain ER2738, purified with polyethylene glycol (PEG) $8,000 / \mathrm{NaCl}$ and subsequently used for the next round of panning. Following two further rounds of panning, single clones were selected randomly from IPTG/ $\mathrm{X}$-gal agar plates and analyzed with a phage enzyme-linked immunosorbent assay (ELISA).

Specific binding of the phage clones to RP215 mAb by phage ELISA. ELISA plates were coated with $50 \mu \mathrm{l}(2 \mu \mathrm{g} / \mathrm{ml}) \mathrm{RP} 215$ as a capture antibody. 4D11, an unrelated $\mathrm{mAb}$, served as an isotype control. Phage particles $\left(1 \times 10^{7}\right.$ plaque-forming units/ml, $50 \mu \mathrm{l} /$ well) from each purified phage clone were added to the plates; an irrelevant phage clone [Advanced Oxidation Protein Products (AOPP)-related phage clone] obtained from colleagues (15) and dilution buffer were used as control groups. The unbound phage particles were washed away with PBST. HRP-labeled anti-M13 (MEK13 phage vector, diluted 1:5,000 with PBST) was added as a detection antibody. The color reaction was developed using tetramethylbenzidine (TMB) with hydrogen peroxide and terminated with $2 \mathrm{M} \mathrm{H}_{2} \mathrm{SO}_{4}$. Subsequently, the absorbance values were read at $450 \mathrm{~nm}$ using a microplate reader (BioTek Instruments, Inc., Winooski, VT, USA). 
Sequencing of the phage clones. Positive phage clones were amplified and purified with a $\mathrm{PEG} / \mathrm{NaCl}$ solution, and the single-stranded DNA was isolated with sodium iodide. Following examination by agarose gel electrophoresis, the single-stranded DNA was sequenced by Invitrogen; Thermo Fisher Scientific, Inc. (Waltham, MA, USA).

Synthesis and characterization of the CA215C peptide mimics Peptide synthesis. Hybio Pharmaceutical Co., Ltd. synthesized three biotinylated single-chain peptides, R2, R13 and R42, which were derived from phage clones 2, 13 and 42, respectively. The accuracy and purity of the final products were confirmed by mass spectrometry and high-performance liquid chromatography, which demonstrated the correct sequence and $90 \%$ purity.

Antigenicity determination by ELISA. Streptavidin was diluted to $5 \mu \mathrm{g} / \mathrm{ml}$ with PBS and immobilized onto microplates. The biotinylated peptides R2, R13 and R42 and the unrelated peptide mimic P39-1 (14) were then serially diluted with PBST, added to the microplates and incubated for $2 \mathrm{~h}$ at room temperature. Subsequently, unbound peptides were washed off with PBST, $1 \mu \mathrm{g} / \mathrm{ml} \mathrm{RP} 215$ or control mouse IgG was added to the microplates and incubated for $30 \mathrm{~min}$ and plates were washed with PBST. Finally, peroxidase AffiniPure goat anti-mouse IgG diluted 1:5,000 with PBST was added, and the color was developed using TMB plus hydrogen peroxide.

To determine whether there were common epitopes between the biotinylated peptides and the human $\mathrm{IgG}, 2 \mu \mathrm{g} / \mathrm{ml}$ goat-anti-human $\mathrm{IgG}$ was immobilized on the microplates as the capture antibody. The biotinylated peptides R 2 and R42 and human $\mathrm{IgG}$ as a positive control were added onto the microplates and incubated at $37^{\circ} \mathrm{C}$ for $30 \mathrm{~min}$. Following washing with PBST, streptavidin-HRP or peroxidase AffiniPure goat anti-mouse IgG diluted 1:5,000 was added to develop a color reaction with $\mathrm{TMB}$.

Immunization of mice with peptide-bovine serum albumin (BSA) conjugates. Initially, peptide mimics were cross-linked with BSA, which acted as a carrier protein. Each of the peptide mimics ( $2 \mathrm{mg}$ ) was diluted with $500 \mu 10.1 \mathrm{M} 2$-(N-Morpholino) ethanesulfonic acid monohydrate (MES) solution ( $\mathrm{pH} 4.5)$; in addition, $4 \mathrm{mg}$ BSA was diluted with MES solution. Peptide (500 $\mu \mathrm{l}$ ) and $400 \mu \mathrm{l}$ BSA were mixed together, and $100 \mu \mathrm{l}$ of freshly prepared 1-(3-dimethylaminopropyl)-3-ethylcarbodiimide solution $\left[10 \mathrm{mg} / \mathrm{ml}\right.$ in double distilled $(\mathrm{dd}) \mathrm{H}_{2} \mathrm{O}$ ] was added to the peptide-BSA mixture, which was then incubated for $4 \mathrm{~h}$ at room temperature with gentle agitation. Finally, the peptide-BSA mixture was dialyzed with $\mathrm{ddH}_{2} \mathrm{O}$ for $48 \mathrm{~h}$ at $4^{\circ} \mathrm{C}$. The conjugates were named R2-BSA/R42-BSA. A total of $15 \mathrm{BALB} / \mathrm{c}$ mice were divided randomly into three groups, and each group was immunized with BSA, R2-BSA or R42-BSA. The immunogens were emulsified with Freund's complete adjuvant and administered via subcutaneous injection for the first immunization. A total of 4 weeks later, five more immunization boosters were performed with Freund's incomplete adjuvant by intraperitoneal injection, with one injection every 2 weeks. The six total immunizations in all of the groups were performed with $100 \mu \mathrm{g}$ immunogen. Finally, antiserum was collected from the tail veins of anesthetized mice and stored at $-20^{\circ} \mathrm{C}$.

Mouse antiserum identification. CA215C binding to murine antisera was detected using ELISA. CA215 $(2 \mu \mathrm{g} / \mathrm{ml}$, $50 \mu \mathrm{l} /$ well) was coated onto microplates and normal human IgG served as a control. Following blocking with casein solution, the antisera, diluted 1:400 in PBST, were added to the microplates (50 $\mu \mathrm{l}$ per well) and incubated for $30 \mathrm{~min}$ at $37^{\circ} \mathrm{C}$. Peroxidase AffiniPure goat anti-mouse IgG antibody diluted 1:5,000 in PBST was then added to the microplates as a detection antibody. Color was developed with TMB and the reaction terminated with $2 \mathrm{M} \mathrm{H}_{2} \mathrm{SO}_{4}$. The absorbance values were read at $450 \mathrm{~nm}$ on a microplate reader.

IHC was performed using antisera on patient samples as previously described by Lee et al (8). Antisera were purified with octylic acid ammonium sulfate precipitation method and the concentration was determined using a UV spectrophotometer. Normal goat serum was used to block any non-specific binding. The following antibodies were diluted in PBS with $2 \% \mathrm{BSA}$ and incubated on ice for $20 \mathrm{~min}: 5 \mu \mathrm{g} / \mathrm{ml} \mathrm{RP} 215$ (positive control), $5 \mu \mathrm{g} / \mathrm{ml} 2 \mathrm{H} 4$ (negative/isotype control), $30 \mu \mathrm{g} / \mathrm{ml}$ anti-R2/R42-BSA antisera and $30 \mu \mathrm{g} / \mathrm{ml}$ anti-BSA antisera (carrier protein control). These were then added to the slides and incubated in a humidified chamber overnight at $4^{\circ} \mathrm{C}$. Peroxidase AffiniPure goat anti-mouse $\operatorname{IgG}(0.8 \mu \mathrm{g} / \mathrm{ml}$ in PBS with $2 \% \mathrm{BSA}$ ) was added to each slide for $40 \mathrm{~min}$ at $37^{\circ} \mathrm{C}$. Finally, staining of the tissue sections was performed with an enhanced HRP-DAB chromogenic substrate kit. The sections were counterstained with hematoxylin and visualized under a light microscope (Olympus Corporation, Tokyo, Japan).

Competitive inhibition of peptide-BSA antisera. Endogenous biotin from the cancer tissue was blocked with an endogenous biotin-avidin blocking kit. Normal goat serum was added to the slides and incubated for $2 \mathrm{~h}$ at room temperature to prevent any non-specific binding. Anti-peptide-BSA antisera $(30 \mu \mathrm{g} / \mathrm{ml})$ and anti-BSA antisera $(30 \mu \mathrm{g} / \mathrm{ml})$ in PBS with $2 \%$ BSA were incubated on ice for $20 \mathrm{~min}$ and then added to the slides as the experimental and negative control groups, respectively. Slides were incubated in a humidified chamber overnight at $4^{\circ} \mathrm{C}$. Subsequently, the slides were brought to room temperature and washed four times with PBS at 5-min intervals on a shaker. Biotinylated RP215 $(5 \mu \mathrm{g} / \mathrm{ml}$ in PBS with $2 \% \mathrm{BSA}$ ) was then added to each slide except for the blank control and incubated for $30 \mathrm{~min}$ at $37^{\circ} \mathrm{C}$. The slides were washed as previously, and incubated with $3 \% \mathrm{H}_{2} \mathrm{O}_{2}$ in PBS at room temperature for $10 \mathrm{~min}$ to remove any endogenous peroxidase activity. The slides were washed three times with PBS at 5-min intervals. Streptavidin-HRP $(1 \mu \mathrm{g} / \mathrm{ml}$ in PBS with $2 \%$ BSA) was added to each slide for $20 \mathrm{~min}$ at $37^{\circ} \mathrm{C}$. Slides were washed as previously, and staining was performed using an enhanced HRP-DAB chromogenic substrate kit. Sections were counterstained with hematoxylin and visualized under a light microscope.

Statistical analysis. Data are expressed as the mean \pm standard deviation. Data were analyzed using two-tailed $t$-tests, using GraphPad Prism software version 6 (GraphPad Software, 
Inc., La Jolla, CA, USA). $\mathrm{P}<0.05$ was considered to indicate a statistically significant difference.

\section{Results}

Phage clones bound to RP215. Following the third round of screening with the anti-CA215 mAb, RP215, 50 blue phage clones were picked from the IPTG/X-gal agar plates. To identify the specificity of the phage clones, RP215 was used as a capture antibody, and 4D11, an unrelated $\mathrm{mAb}$, served as a control antibody. In total, 33 phage clones demonstrated strong binding to RP215 without binding to 4D11 (Fig. 1), whereas irrelevant control, AOPP-related phage and blank control did not bind to RP215, suggesting that the displayed peptides on these 33 positive phage clones may be complementary epitopes to RP215. This experiment was performed three times, and the 30 positive phage clones with the greatest strength and stability of binding were selected for sequencing.

Sequence analysis of phage clones. In total, 30 positive phage clones were amplified to obtain purified DNA for sequencing. A total of 23 sequences were analyzed, as certain phage clones demonstrated identical sequences. Phage clones 1, 2, 5, 6, 11 and 31 shared the conserved E-LWR sequence (sequence I); clones 4, 12, 25 and 36 shared the E-HWR sequence (sequence II); clones 18, 21 and 39 phage contained the E-WR sequence (sequence III); phage clones 3 and 40 shared the EDLW conserved sequence (sequence IV); and phage clones 7 and 42 possessed the conserved E-LWR(K) sequence (sequence $\mathrm{V}$ ). There were no conserved sequences in the remaining the phage clones (Table I). The sequence E-(-)WR was present in four conserved sequence groups, although the amino acids between amino E and WR were not identical. Furthermore, phage clones 2 (LSTKEVESLWRR), 42 (HKASWERDLWRE) and 13 (ISEKQVESLRRR) had high-affinity binding to low RP215 concentrations (Fig. 2). Consequently, the peptide synthesis reactions were performed based on the sequences of clones 2, 42 and 13 .

Antigenicity of the biotinylated CA215C peptide mimics. To identify the antigenicity of peptide mimics, short peptides were synthesized in the form of biotinylated peptides, and binding to RP215 was assessed by ELISA. The biotinylated peptides R2 and R42 bound to RP215 in a dose-dependent manner, whereas the R13 biotinylated peptide and an unrelated peptide, P39-1 (14), did not (Fig. 3A). Additionally, no cross-reaction of the biotinylated peptides R2, R13 and R42 with normal mouse IgG was observed (Fig. 3B). Furthermore, there was no cross-reaction between the biotinylated peptides R2 and R42 and goat-anti-human IgG (Fig. 3C). These results indicated that CA215C peptide mimics with the conserved sequences from R2 and R42 demonstrated antigenicity with RP215, which suggested that the biotinylated peptides R2 and R42 may mimic the CA215C binding epitope of RP215.

Immunogenicity of $R 2$ and $R 42$. As the molecular weights of the short peptides were too low to directly use in immunization without a carrier, biotinylated peptides R2 and R42 were conjugated to BSA, which served as a carrier protein. The
Table I. Amino acid sequences of phage clones that bound to RP215.

Phage clones ${ }^{\mathrm{a}}$

Amino acid sequences ${ }^{\mathrm{b}}$

$\begin{array}{ll}1,2,5,6,11,31 & \text { E-LWR }^{\mathrm{c}} \\ 4,12,25,36 & \text { E-HWR }^{\mathrm{c}} \\ 18,21,39 & \text { E-WR }^{\mathrm{c}} \\ 3,40 & \text { EDLW } \\ 7,42 & \text { E-LWR(K) } \\ 13,15,19,32,33,35 & \text { Irregular }\end{array}$

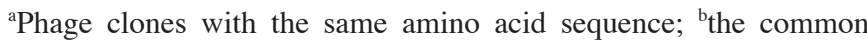
sequence in each group; ${ }^{\mathrm{c}}$-represent heterogenous amino acids in the sequences of phage clones.

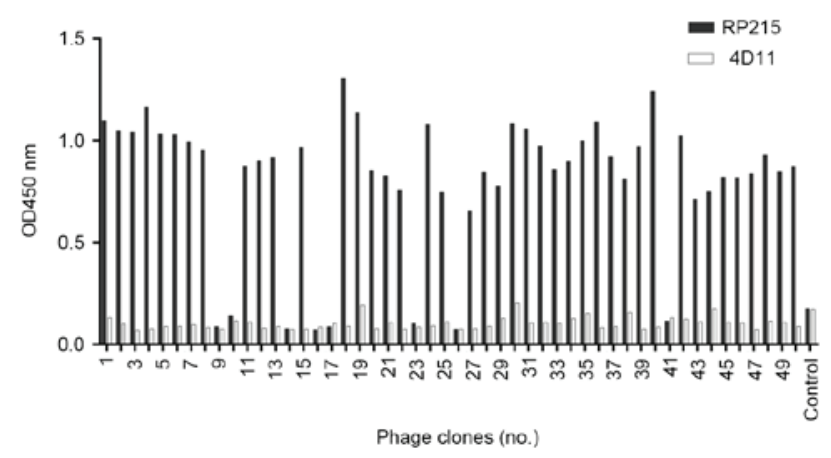

Figure 1. Binding of 50 phage clones to RP215. ELISA was performed to determine the binding of the 50 phage clones to RP215 (black bars) and an unrelated mAb, 4D11 (white bars). A total of 33 phage clones demonstrated strong binding to RP215 without binding to 4D11.

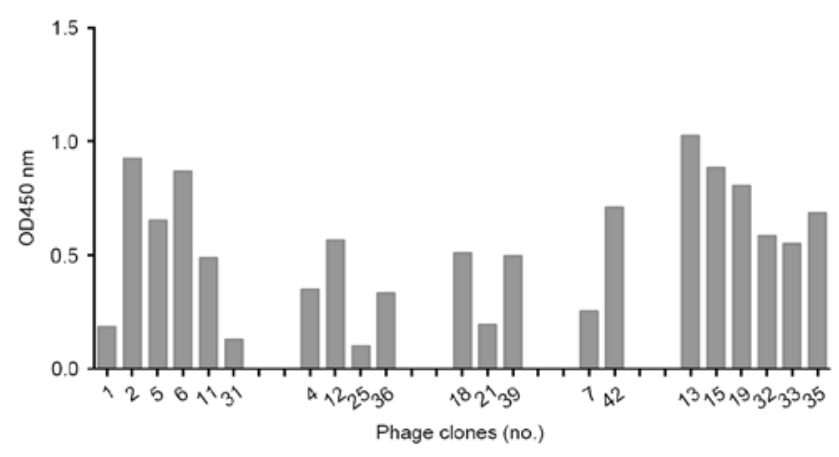

Figure 2. Binding of phage clones with various conserved sequences to low concentration RP215. Each phage clone was adjusted to the same titer and binding to RP215 was assessed by ELISA. Phage clones 1, 2, 5, 6, 11 and 31 had the E-LWR conserved sequence; phage clones 4, 12, 25, and 36 possessed the E-HWR sequence, phage clones 18, 21, and 39 possessed the E-WR sequence; and phage clones 7 and 42 had the E-LWR(K) sequence. The sequences in phage clones 13, 15, 19, 32, 33 and 35 were irregular. Phage clones 2, 42 and 13 had high-affinity binding to low RP215 concentrations.

immunogenicity of R2 and R42 short peptides was confirmed by immunizing BALB/c mice with R2-BSA or R42-BSA conjugates, while immunization with BSA alone served as a control. The antisera of R2-BSA $(\mathrm{P}<0.0001)$ and R42-BSA $(\mathrm{P}=0.0005)$-immunized mice reacted with $\mathrm{CA} 215 \mathrm{C}$, whereas 

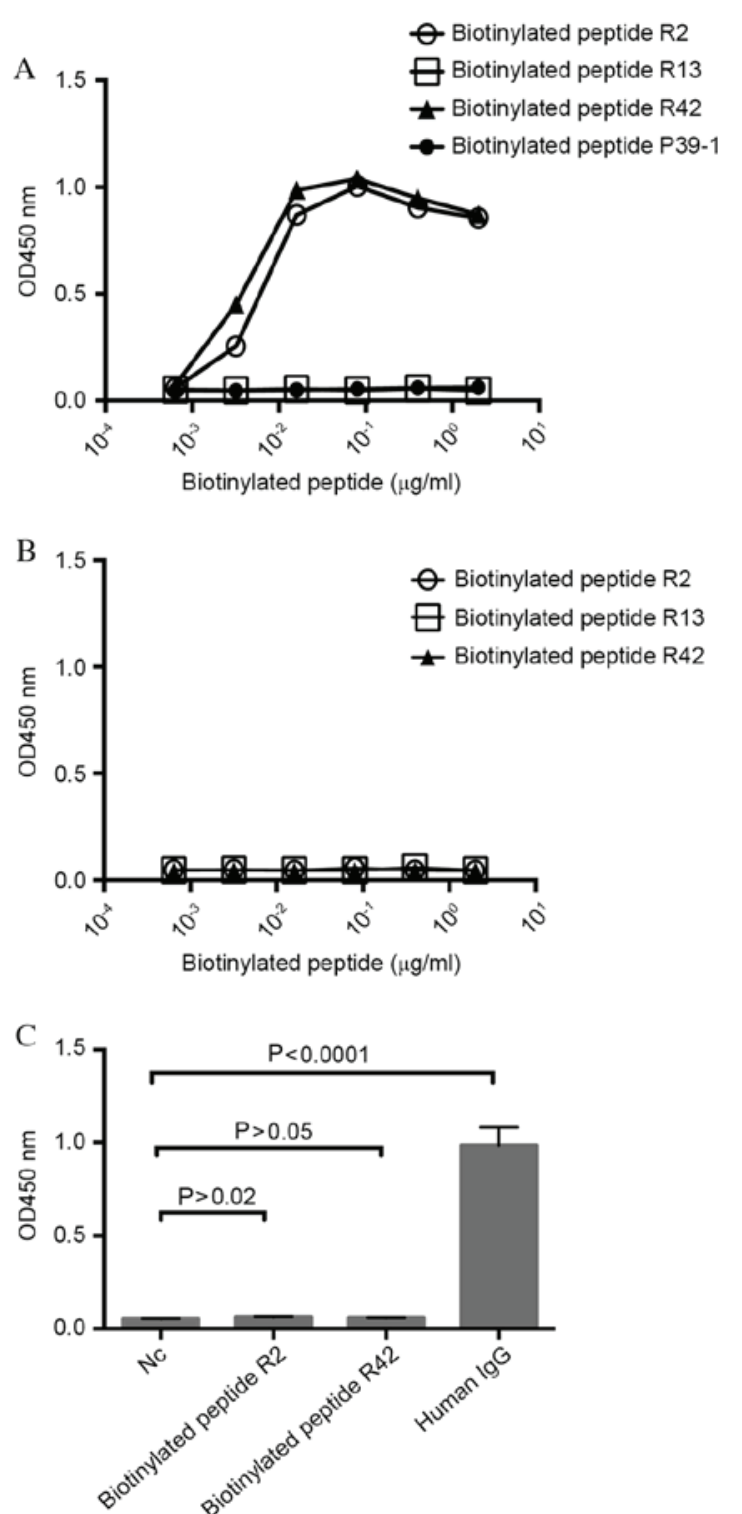

Figure 3. Specific binding of the biotinylated peptides, R2, R13 and R42 to RP215, as assessed by ELISA. The biotinylated peptides were bound to streptavidin-coated microwells at serial dilution, followed by addition of (A) RP215 or (B) control mouse IgG antibody. (C) The biotinylated peptides were bound to goat-anti-human $\mathrm{IgG}$-coated microwells. Dilution buffer served as a negative control, and human IgG served as a positive control. Data are expressed as the mean \pm standard deviation. $\mathrm{Nc}$, negative control.

there was no significant binding to normal human $\operatorname{IgG}$ (R2-BSA antisera, $\mathrm{P}=0.3660$; R42-BSA antisera, $\mathrm{P}=0.1601$; Fig. 4). The murine antisera were then reacted with the native CA215C epitope expressed in human tumor tissues, by IHC. As for the RP215 positive control (Fig. 5A-a), antisera from mice immunized with R2-BSA (Fig. 5A-b) or R42-BSA (Fig. 5A-c) bound to human hepatic carcinoma tissue, whereas no binding was observed using antisera from mice immunized with BSA only (Fig. 5A-d), using the irrelevant mAb, 2H4 (Fig. 5A-e) or in the blank control (Fig. 5A-f). Similar results were observed in rectal carcinoma tissue (Fig. 5B). Furthermore, murine antisera inhibited RP215 binding to tumor tissue, including hepatic (Fig. 6A) and rectal (Fig. 6B) carcinoma tissue, compared with controls. These results
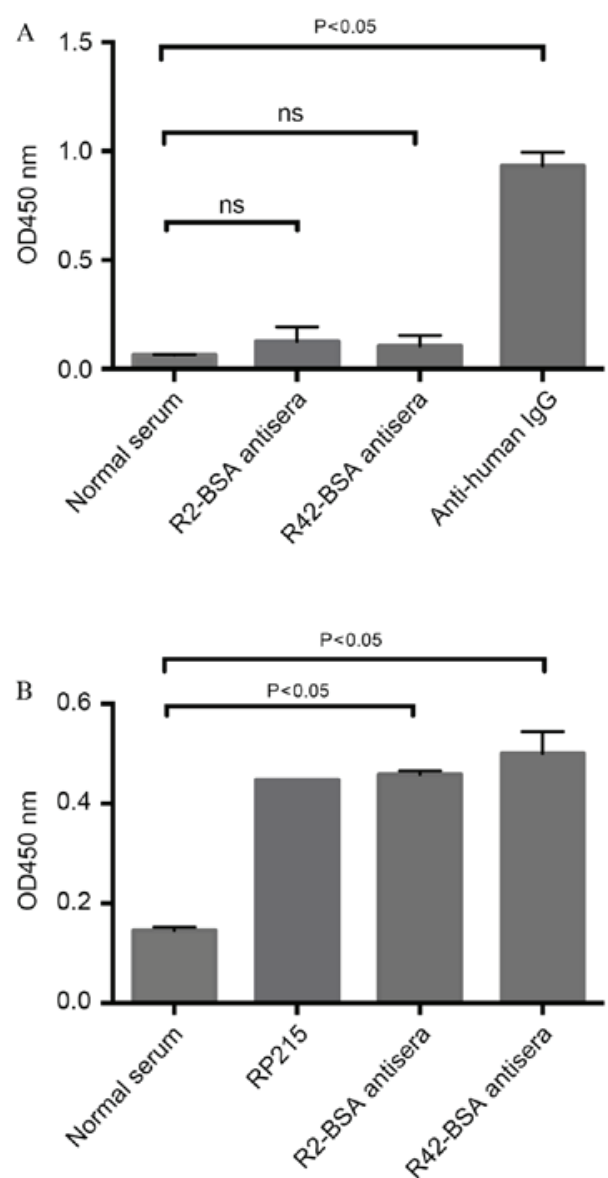

Figure 4. Specific binding of R2-BSA and R42-BSA antisera to CA215C, as assessed by ELISA. R2-BSA and R42-BSA antisera, were added to (A) human IgG-coated microwells or (B) CA215-coated microwells (A) Anti-human IgG and (B) RP215 served as positive controls. Normal serum served as a negative control. The antisera of R2-BSA and R42-BSA immunized mice reacted with CA215C, whereas there was no significant binding to normal human IgG. Data are expressed as the mean \pm standard deviation. ns, non-significant.

indicated that $\mathrm{R} 2$ and $\mathrm{R} 42$ peptides mimicked the CA215C epitope characteristics and induced serum antibodies with activities similar to RP215 in mice.

\section{Discussion}

In the present study, short peptide mimics of a carbohydrate-associated epitope of a tumor antigen were successfully obtained by screening a random phage display library with the specific $\mathrm{mAb}, \mathrm{RP} 215$. In addition, these peptide mimics induced the production of antisera that could bind to CA215C, suggesting that these short peptides mimicked the CA215C epitope. Furthermore, the results indicated that the carbohydrate epitope, a TI-Ag, was altered to a peptide epitope that may become a thymus-dependent antigen (TD-Ag) by carrier protein conjugation or formation of multiple antigen peptides. In the past two decades, the importance of carbohydrate-associated epitopes of pathogen and tumor antigens has been increasingly investigated, as numerous studies have demonstrated that carbohydrate or glycolipid structures or epitopes on pathogens and tumor cells are important as potential biomarkers or vaccine candidates, 

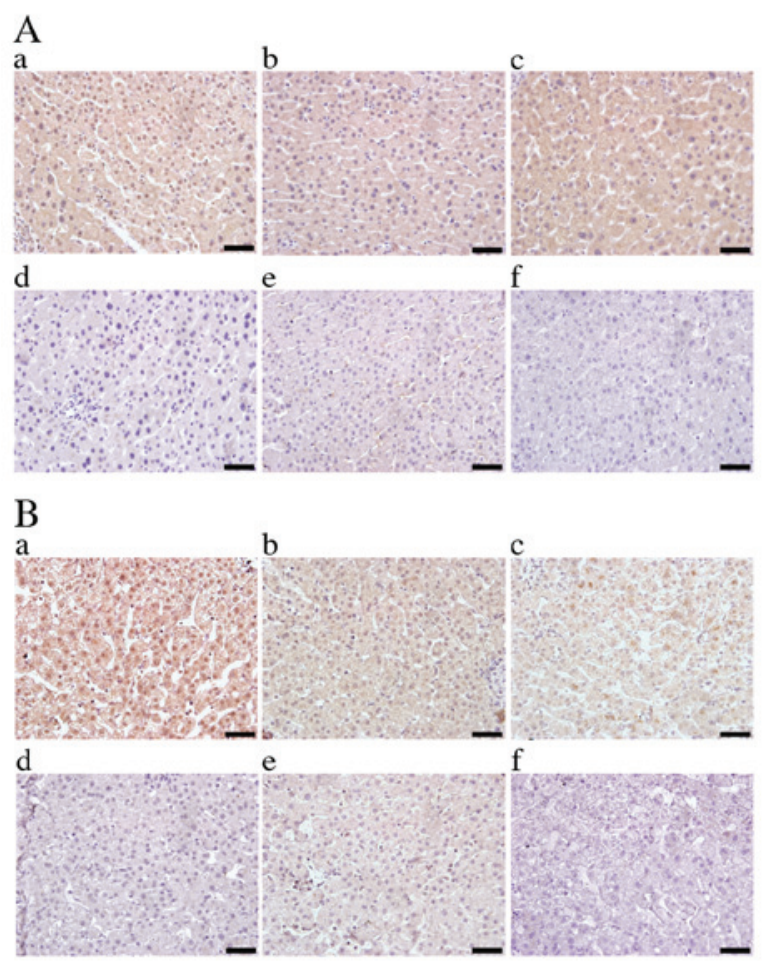

Figure 5. Specific binding of R2-BSA and R42-BSA antisera to patient tissue samples, as assessed by immunohistochemistry. (A) Hepatic and (B) rectal carcinoma tissues were stained with (a) RP215 as a positive control, (b) anti-R2-BSA antisera, (c) anti-R42-BSA antisera, (d) anti-BSA antisera, (e) the unrelated $\mathrm{mAb}, 2 \mathrm{H} 4$, as a negative control and (f) a blank control. Antisera from mice immunized with R2-BSA or R42-BSA bound to human hepatic and rectal carcinoma tissue. Scale bars $=50 \mu \mathrm{m}$.

including LPS (16) and Thomsen-Friedenreich antigen (Galß1-3GalNAc $\alpha$-O-Ser/Thr) (17). However, there are two challenges for the application or development of vaccine candidates for carbohydrate or glycolipid epitopes: i) These types of epitope are difficult to obtain by biological purification or recombinant expression and ii) the characteristics of a TI-Ag render them incapable of inducing effective secondary antibody responses and cellular immunity. As an alternative strategy, peptide mimics structurally simulate non-protein epitopes and may alter them from TI-Ag to TD-Ag. In addition, peptide mimics may be readily synthesized and conjugated.

Prior to the present study regarding CA215C peptide mimics, numerous studies have successfully generated peptide mimics. For example, Monzavi-Karbassi et al (18) reported that a peptide surrogate of GlcNAc induced an in vivo tumor-specific cellular response to established Meth A tumors that expressed native O-GlcNAc glycoproteins on the tumor cell surface. In addition, peptide mimics of pathogens have been generated, particularly for non-protein elements, including LPS (16) and peptidoglycan (14). Furthermore, peptide-mimic strategies using random peptide libraries have been used for nonlinear or structural epitope analyses $(19,20)$. However, not all synthesized peptides based on screened sequences from peptide libraries exhibit the expected antigenicity, even though positive phage clones or bead-conjugated peptides from combinatory peptide libraries bind well to specific antibodies. For example, it is very difficult to obtain short linear peptides that mimic structural epitopes on protein
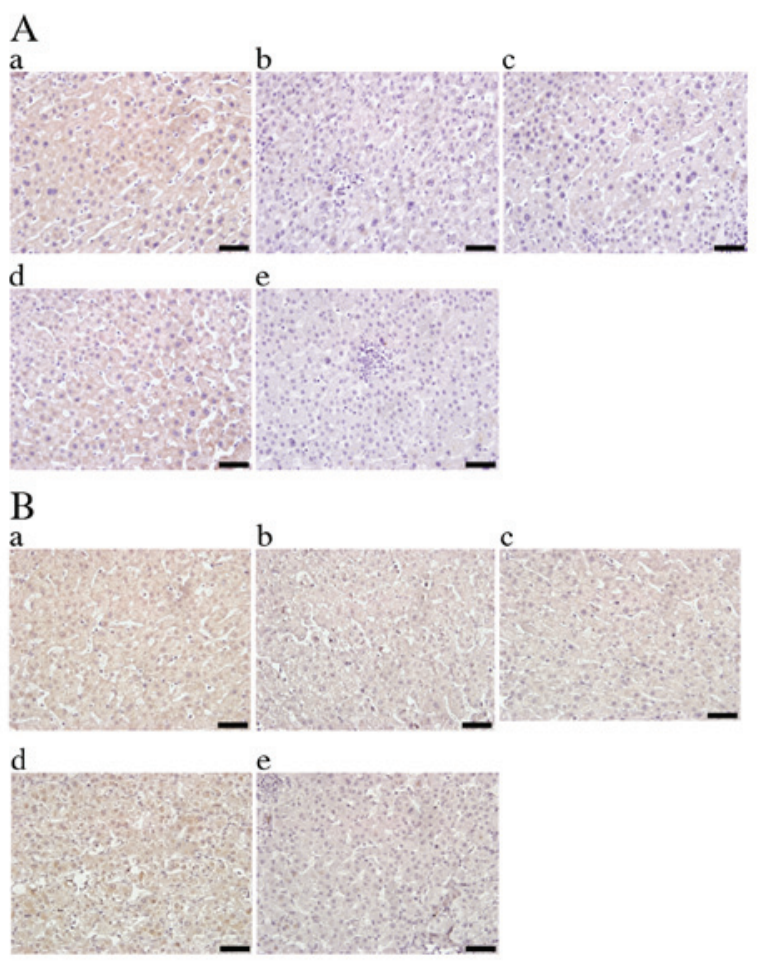

Figure 6. Competitive inhibition study of R2-BSA and R42-BSA antisera in patient samples, as assessed by immunohistochemistry. (A) Hepatic and (B) rectal carcinoma tissues were stained with (a) biotinylated RP215 only, as a positive control, (b) anti-R2-BSA antisera followed by biotinylated RP215, (c) anti-R42-BSA antisera followed by biotinylated RP215, (d) anti-BSA antisera followed by biotinylated RP215 and (e) dilution buffer only as a blank control. Antisera from mice immunized with R2-BSA or R42-BSA inhibited RP215 binding to human hepatic and rectal carcinoma tissue. Scale bars $=50 \mu \mathrm{m}$.

molecules. In addition, numerous short linear peptides have been synthesized based on screened sequences from phage display peptide libraries using antibodies to protein epitopes, that could not bind to antibodies for screening during subsequent determination experiments; however, longer linear or cyclical peptide mimics to non-protein epitopes appear to be antigenic, including peptide mimics to Escherichia coli LPS $(14,16,21)$.

In the present study, two specific and antigenic peptide mimics were obtained of a carbohydrate epitope recognized by the mAb, RP215, which binds CA215C on numerous tumor cell types and inhibits tumor cell growth $(7,10)$. Following three rounds of screening and positive clone identification with sandwich ELISAs that utilized RP215 at a very low concentration, DNA sequencing from 30 positive phage clones was performed. From these results, five conserved sequence groups were obtained: E-LWR, E-HWR, E-WR, EDLW and E-LWR(K); E-WR may serve as a more conserved sequence for four of the groups. According to the affinity of phage clones with RP215, clones 2 (LSTKEVESLWRR), 42 (HKASWERDLWRE) and 13 (ISEKQVESLRRR) were selected for peptide synthesis. The R2 (sequence from clone 2) and R42 (sequence from clone 42)-synthesized peptides bound RP215 at a concentration of $10^{-2} \mu \mathrm{g} / \mathrm{ml}$; however, they did not bind with anti-human $\operatorname{IgG}$, suggesting a high degree of sensitivity and specificity. 
CA215 is homologous with normal human $\operatorname{IgG}$, and at least 32 tryptic peptide fragments exhibited various degrees of homology with human Igs (60-100\%). Although N-linked and O-linked carbohydrate composition analyses demonstrate similar compositions of monosaccharides in normal human IgG and RP215 (mouse $\operatorname{IgG}$ ), CA215 exhibits a distinct sugar content compared with normal human IgG and mouse IgG. In particular, CA215 contains a reduced percentage of $\mathrm{N}$-acetylglucosamine and significantly greater quantities of mannose, $\mathrm{N}$-acetylneuraminic acid and $\mathrm{N}$-glycoylneuraminic acid $(4,22)$. Therefore, the ideal short peptide should mimic the CA215C epitope, a specific carbohydrate-associated epitope on the IgG heavy chain $\mathrm{V}$ region from cancer cells, which is not expressed on normal $\operatorname{IgG}$; in this way the epitope mimicked by the short peptide would not be a common epitope between normal human IgG and CA215. Goat anti-human IgG antibody, a polyclonal antibody that recognizes all epitopes of normal human IgG, was almost incapable of binding to the R2 and R42 synthetic peptide mimics. This result suggested that the R2 and R42 peptides have no shared epitope with normal human IgG and that these synthetic R2 and R42 peptides may mimic a CA215C epitope.

In addition, the present study confirmed that peptide mimics of CA215C may be potential vaccine candidate epitopes by preparing antisera following immunization with peptide mimic conjugates and BSA as a carrier protein. The R2-BSA and R42-BSA peptide mimics reacted with RP215 in a dose-dependent manner; however, they did not react with goat anti-human $\operatorname{IgG}$ polyclonal antibody in an ELISA experiment (data not shown). This result demonstrated that the mimic epitopes for CA215C, specifically recognized by RP215, remained and that the R2-BSA and R42-BSA cross-linked peptides may be used to evaluate the efficacy of these synthetic peptides for activating the immune system.

$\mathrm{BALB} / \mathrm{c}$ mice were separately immunized with the R2-BSA and R42-BSA conjugates and the resultant antisera were analyzed by ELISA and IHC. Murine antisera reacted with CA215 specifically; no significant reaction with normal human or mouse IgG occurred. Notably, murine antisera bound to human hepatic and rectal carcinoma tissue, and this inhibited RP215 binding. Furthermore, the binding of RP215 to cancer tissues, including human hepatic and rectal carcinoma tissue, was inhibited by antisera from mice immunized with R2-BSA or R42-BSA conjugates. These results indicated that the R2 and R42 peptides mimicked a CA215C epitope. Therefore, these synthetic peptides may be utilized as vaccine candidate epitopes to induce specific antibodies against cancer cells through CA215C in humans.

Further investigation is required to develop a viable tumor vaccine from a vaccine candidate or candidate epitope. In future studies, the immunogenicity may be enhanced by exchanging certain amino acids, synthesizing a four-branch multiple antigenic peptide that may not require a carrier protein $(23,24)$ and selecting a suitable adjuvant $(25)$.

In conclusion, the results of the present study demonstrated that R2-BSA and R42-BSA antisera had similar characteristics to RP215 and that the synthetic peptides $\mathrm{R} 2$ and R42 may mimic the CA215C epitope. R2 and R42 peptides may therefore have potential for development into a tumor vaccine.

\section{Acknowledgements}

The authors thank Professor Gregory Lee for donating CA215 and the $\mathrm{mAb}, \mathrm{RP} 215$. The present study was supported in part by the Special Project on the Integration of Industry, Education and Research of Guangdong Province (grant no. \#2012B091000148), and the Nature Science Foundation of Guangdong Province (grant no. 2014A030313281).

\section{References}

1. Qiu X, Zhu X, Zhang L, Mao Y, Zhang J, Hao P, Li G, Lv P, Li Z, Sun $X$, et al: Human epithelial cancers secrete immunoglobulin $\mathrm{g}$ with unidentified specificity to promote growth and survival of tumor cells. Cancer Res 63: 6488-6495, 2003.

2. Li M, Feng DY, Ren W, Zheng L, Zheng H, Tang M and Cao Y: Expression of immunoglobulin kappa light chain constant region in abnormal human cervical epithelial cells. Int J Biochem Cell Biol 36: 2250-2257, 2004.

3. Chen Z, Huang X, Ye J, Pan P, Cao Q, Yang B, Li Z, Su M, Huang C and $\mathrm{Gu} \mathrm{J}$ : Immunoglobulin $\mathrm{G}$ is present in a wide variety of soft tissue tumors and correlates well with proliferation markers and tumor grades. Cancer 116: 1953-1963, 2010.

4. Lee G, Laflamme E, Chien CH and Ting HH: Molecular identity of a pan cancer marker, CA215. Cancer Biol Ther 7: 2007-2014, 2008.

5. Lee G: Cancer cell-expressed immunoglobulins: CA215 as a pan cancer marker and its diagnostic applications. Cancer Biomark 5: 137-142, 2009.

6. Lee G and Ge BX: Cancer cell expressions of immunoglobulin heavy chains with unique carbohydrate-associated biomarker. Cancer Biomark 5: 177-188, 2009.

7. Lee G, Ge B, Huang TK, Zheng G, Duan J and Wang IH: Positive identification of CA215 pan cancer biomarker from serum specimens of cancer patients. Cancer Biomark 6: 111-117, 2010.

8. Lee G, Zhu M, Ge B and Potzold S: Widespread expressions of immunoglobulin superfamily proteins in cancer cells. Cancer Immunol Immunother 61: 89-99, 2012.

9. Lee G, Cheung AP, Li B, Ge B and Chow PM: Molecular and immuno-characteristics of immunoglobulin-like glycoproteins in cancer cell-expressed biomarker, CA215. Immunol Invest 41: 429-446, 2012.

10. Lee $\mathrm{G}$ and Ge B: Inhibition of in vitro tumor cell growth by RP215 monoclonal antibody and antibodies raised against its anti-idiotype antibodies. Cancer Immunol Immunother 59: 1347-1356, 2010.

11. Lee G, Chu RA and Ting HH: Preclinical assessment of anti-cancer drugs by using RP215 monoclonal antibody. Cancer Biol Ther 8: 161-166, 2009.

12. Tang L, Zhu P, Luo C, Xu XM and Fu N: Preparation and identification of monoclonal antibodies against different epitopes on human zeta globin chain. Di Yi Jun Yi Da Xue Xue Bao 25: 1394-1397, 2005 (In Chinese).

13. Xiao H, Zhu P, Liu B, Pan Q, Jiang X, Xu X and Fu N: Generation and characterization of human delta-globin-specific monoclonal antibodies. Blood Cells Mol Dis 44: 127-132, 2010.

14. Chen Y, Liu B, Yang D, Li X, Wen L, Zhu P and Fu N: Peptide mimics of peptidoglycan are vaccine candidates and protect mice from infection with Staphylococcus aureus. J Med Microbiol 60: 995-1002, 2011.

15. Hou XR, Liu BY, Tian JW, Chen YG and Fu N: Screening and Characterization of the Mimotopes of Adveanced Oxidation Protein Products from Phage Peptide Library. Chinese Journal of Laboratory Diagnosis 15: 765-768, 2011.

16. Nagy G and Pál T: Lipopolysaccharide: A tool and target in enterobacterial vaccine development. Biol Chem 389: 513-520, 2008.

17. Samuel J, Noujaim AA, MacLean GD, Suresh MR and Longenecker BM: Analysis of human tumor associated Thomsen-Friedenreich antigen. Cancer Res 50: 4801-4808, 1990.

18. Monzavi-Karbassi B, Luo P, Jousheghany F, Torres-Quiñones M, Cunto-Amesty G, Artaud C and Kieber-Emmons T: A mimic of tumor rejection antigen-associated carbohydrates mediates an antitumor cellular response. Cancer Res 64: 2162-2166, 2004. 
19. Tiwari R, Negi SS, Braun B, Braun W, Pomés A, Chapman MD, Goldblum RM and Midoro-Horiuti T: Validation of a phage display and computational algorithm by mapping a conformational epitope of Bla g 2. Int Arch Allergy Immunol 157: 323-330, 2012.

20. Gazarian KG, Palacios-Rodriguez Y, Gazarian TG and Huerta L: HIV-1 V3 loop crown epitope-focused mimotope selection by patient serum from random phage display libraries: Implications for the epitope structural features. Mol Immunol 54: 148-156, 2013.

21. Hossany RB, Johnson MA, Eniade AA and Pinto BM: Synthesis and immunochemical characterization of protein conjugates of carbohydrate and carbohydrate-mimetic peptides as experimental vaccines. Bioorgan Med Chem 12: 3743-3754, 2004.
22. Lee G and Azadi P: Peptide mapping and glycoanalysis of cancer cell-expressed glycoproteins CA215 recognized by RP215 monoclonal antibody. J Carbohyd Chem 31: 10-30, 2012

23. Wu YZ, Zhang JB, Chen SY, Chen A, Wang L, Li J, Zhao T, Zou L, Tang Y, Tingrong L and Wang F: Frequencies of epitope-specific cytotoxic T lymphocytes in active chronic viral hepatitis B infection by using MHC class I peptide tetramers. Immunol Lett 92: 253-258, 2004

24. Yamada A, Sasada T, Noguchi M and Itoh K: Next-generation peptide vaccines for advanced cancer. Cancer Sci 104: 15-21, 2013.

25. Cheng WK, Wee K, Kollmann TR and Dutz JP: Topical CpG adjuvantation of a protein-based vaccine induces protective immunity to Listeria monocytogenes. Clin Vaccine Immunol 21: 329-339, 2014. 